\title{
Corrigendum: Insulator Contamination Perception Based on Feature Fusion of Infrared Image and Meteorological Parameters
}

\author{
Hongxia Wang ${ }^{1}$, Bo Wang ${ }^{1 \star}$, Min $\mathrm{Li}^{2}$, Peng Luo ${ }^{1}$, Hengrui Ma ${ }^{3}$ and Fuqi Ma ${ }^{1}$ \\ ${ }^{1}$ School of Electrical Engineering and Automation, Wuhan University, Wuhan, China, ${ }^{2}$ School of Computer and Artificial \\ Intelligence, Wuhan Textile University, Wuhan, China, ${ }^{3}$ New Energy (Photovoltaic) Industry Research Center, Qinghai University, \\ Xining, China
}

Keywords: insulator, contamination perception, feature fusion, infrared image, meteorological parameters

\section{A Corrigendum on}

Insulator Contamination Perception Based on Feature Fusion of Infrared Image and Meteorological Parameters

by Wang, H., Wang, B., Li M., Luo, P., Ma, H., and Ma, F. (2021). Front. Energy Res. 9:746378. doi: 10. 3389/fenrg.2021.746378

\section{OPEN ACCESS}

Edited and reviewed by: Xun Shen,

Tokyo University of Agriculture and Technology, Japan

*Correspondence: Bo Wang

whwdwb@whu.edu.cn

Specialty section: This article was submitted to Wind Energy,

a section of the journal Frontiers in Energy Research

Received: 09 September 2021 Accepted: 17 September 2021 Published: 18 October 2021

Citation:

Wang $H$, Wang B, Li M, Luo P, Ma H and $M a F$ (2021) Corrigendum: Insulator Contamination Perception Based on Feature Fusion of Infrared Image and Meteorological Parameters.

Front. Energy Res. 9:773322.

doi: 10.3389/fenrg.2021.773322
In the original article, there was an error. In the description of Eq. 4, the last part of the sentence that reads "which equals to 1,000 in the VGG-16 and 5 in the feature fusion network proposed." should be removed.

In addition, there was also an error in section 4 Experimental Setups, 4.2 Training Phase. The final sentence was incorrectly written as "where $L$ represents the loss; pi is the probability from softmax, ppi represents the label of the input, which is the real class of the input data; $n$ is the number of samples, which equals to 1,000 in the VGG-16 and 5 in the feature fusion network proposed." The sentence should read as follows: "where $L$ represents the loss; $p_{i}$ is the probability from softmax, $p_{i}^{*}$ represents the label of the input, which is the real class of the input data; $n$ is the number of samples."

Finally, there was also an error in the Funding statement. The original statement was incorrectly written as "This paper is supported by the National Natural Science Foundation of China (Grant No.51777142,51907096)". The statement should read as follows: "This paper is supported by the State Grid Headquarters Technology Project (Grant No.5400-202119145A-0-0-00)”.

The authors apologize for this error and state that this does not change the scientific conclusions of the article in any way. The original article has been updated.

Conflict of Interest: The authors declare that the research was conducted in the absence of any commercial or financial relationships that could be construed as a potential conflict of interest.

Publisher's Note: All claims expressed in this article are solely those of the authors and do not necessarily represent those of their affiliated organizations, or those of the publisher, the editors and the reviewers. Any product that may be evaluated in this article, or claim that may be made by its manufacturer, is not guaranteed or endorsed by the publisher.

Copyright $(2021$ Wang, Wang, Li, Luo, Ma and Ma. This is an open-access article distributed under the terms of the Creative Commons Attribution License (CC BY). The use, distribution or reproduction in other forums is permitted, provided the original author(s) and the copyright owner(s) are credited and that the original publication in this journal is cited, in accordance with accepted academic practice. No use, distribution or reproduction is permitted which does not comply with these terms. 